Galán, S. La Computación Cuántica: nuevos desafíos para el sistema legal argentino Derecho y Ciencias Sociales. Noviembre 2020 - Abril 2021. ํo 24 .Pgs 123 -135 ISSN 1852-2971. Instituto de Cultura Jurídica y Maestría en Sociología Jurídica. Facultad de Ciencias Jurídicas y Sociales. Universidad Nacional de La Plata. Argentina.

\title{
La Computación Cuántica: nuevos desafíos para el sistema legal argentino
}

Quantum Computing: new challenges for the Argentine legal system

Santiago Galán•

\section{Resumen}

En el presente estudio me propongo ahondar en las nuevas irrupciones generadas por la Computación Cuántica; el objetivo perseguido con el mismo es dar a conocer y entender de qué se trata la misma, al punto de poder explicar que efectos genera o puede generar para distintas empresas y países en general. Dicho conocimiento considero de gran valor para el universo de las ciencias jurídicas, en el cual, se deben plantear y adaptar las normas de todos los países del mundo. Especialmente considero que las normas de la Argentina requieren de una renovación de manera urgente, con el objetivo de optimizar los beneficios que pueda traer la misma y reducir los conflictos que de ella puedan surgir.

Palabras Clave: Computación Cuántica, datos personales, ley, protección

\begin{abstract}
In the present study I propose to delve into the new eruptions generated by Quantum Computing; the objective pursued with it is to make known and understand what it is about, to the point of being able to explain what effects it generates or can generate for different companies and countries in general. This knowledge I consider of great value to the universe of legal sciences, in which, the norms of all the countries of the world must be raised and adapted. I especially consider that Argentina's regulations urgently require renewal, with the aim of optimizing the benefits that it may bring and reducing the conflicts that may arise from it.
\end{abstract}

Keywords: Quantum Computing, personal data, law, protection

\footnotetext{
- Abogado graduado de la Facultad de Ciencias Jurídicas y Sociales de la Universidad Nacional de La Plata. Maestrando en Derechos Humanos de la Maestría de Derechos Humanos de la Universidad Nacional de La Plata. Actualmente cursando Diplomatura de "Derecho 4.0" de la Universidad Austral. Auxiliar en la Unidad de Investigación de Delitos Económicos de Policía Judicial; del Departamento de Política Criminal de la Procuración de la Suprema Corte de Justicia de Buenos Aires. Contacto: sgalan@live.com.ar
} 
Galán, S. La Computación Cuántica: nuevos desafíos para el sistema legal argentino Derecho y Ciencias Sociales. Noviembre 2020 - Abril 2021. ํo 24 .Pgs 123 -135 ISSN 1852-2971. Instituto de Cultura Jurídica y Maestría en Sociología Jurídica. Facultad de Ciencias Jurídicas y Sociales. Universidad Nacional de La Plata. Argentina.

\section{La Computación Cuántica: nuevos desafíos para el sistema legal argentino}

Santiago Galán

\section{Introducción}

En el presente artículo me propongo hacer una reseña sobre un avance en las ciencias de la computación que se encuentra en pleno desarrollo. El mismo ya está teniendo efectos en distintas ramas de la ciencia, e inquieta a las principales empresas a nivel mundial en alcanzarlo a niveles óptimos. Así también conlleva un fuerte avance en industrias que nacieron en los últimos años y como consecuencia lógica el mundo del derecho no podrá estar ajeno al mismo. Dicha tecnología se denomina computación cuántica ${ }^{1}$.

Para abordar la computación cuántica uno debe indagar en el mundo de las ciencias de la computación como así también en las ciencias de la física, las cuales bien sabemos son ajenas al derecho. Sin embargo, la propuesta no pasa por dar explicaciones extensas desde el punto de vista técnico de la cuestión, dicha situación más bien es abordada de forma muy laxa, ya que lo fundamental aquí se trata de entender la potencialidad de los efectos que trae consigo la computación cuántica (Darwish Miranda, 2020:7).

En efecto, al detenernos en la potencialidad que exhibe en el presente y hacia el futuro dicho avance, podremos entender la relevancia de abordar este fenómeno de antemano a su producción en masa y así prepararnos -desde el lugar que nos toca- para poder tratar la cuestión de forma provechosa, a los fines de no lamentar ni dejar pasar una oportunidad que promete ser histórica para la tecnología tal y como la conocemos.

Cabe decir que la misma se encuentra siendo desarrollada por las principales empresas tecnológicas a nivel mundial, como así también por los Estados que detentan más poderío científico y económico, en vías de una suerte de competencia por ver quién puede ostentar primero su producción a gran escala y explotación.

\footnotetext{
${ }^{1}$ Antes de comenzar a hablar de la computación cuántica tal vez sea importante señalar que hay mucho más que decir sobre este tema que simplemente ceñirnos a una implementación particular de una máquina para hacer cálculos. El computador cuántico es una idea que crece a medida que se desarrolla la teoría cuántica de la información. De este modo cada una de estas entidades crece con ayuda de la otra. Lo cierto es que a medida que vayamos afrontando los problemas que surgen a la hora de crearlo, aprenderemos más y más sobre cómo funcionan los sistemas cuánticos y sobre cómo se comporta la información que de ellos somos capaces de obtener"
} 
Galán, S. La Computación Cuántica: nuevos desafíos para el sistema legal argentino Derecho y Ciencias Sociales. Noviembre 2020 - Abril 2021. ํo 24 .Pgs 123 -135 ISSN 1852-2971. Instituto de Cultura Jurídica y Maestría en Sociología Jurídica. Facultad de Ciencias Jurídicas y Sociales. Universidad Nacional de La Plata. Argentina.

\section{¿De qué se trata la computación cuántica?}

Primero es menester explicar brevemente de que se trata la computación cuántica, para ello brindare una somera noción científica al respecto, que servirá para entender en primer término sus aptitudes y capacidades de cálculo. Luego pasare a mencionar los usos para los cuales está pensando en la actualidad por distintos científicos, posteriormente brindare un análisis estrictamente jurídico de dicho asunto, en el cual, explicare mi postura sobre la urgencia de brindar debates, tanto a nivel académico como de postular distintos modos de regular la misma. Finalmente describiré ciertos proyectos de los cuales se están encargando los Estados más poderosos, algunos de los cuales ya brindan frutos en la actualidad y otros que aún deben esperar.

La Computación Cuántica ${ }^{2}$ surge al combinar la teoría cuántica de la física, en forma conjunta a la ciencia que estudia la computación. En la computación clásica que conocemos popularmente, utilizamos unidades de información llamadas bits, en cambio en la computación cuántica las unidades mínimas de información que utilizaremos serán cubits. La diferencia sustancial entre los bits y los cubits radica tanto en la capacidad como en la velocidad de transmitir información. En términos de computación digital, esto quiere decir que mientras un bit solo puede tomar dos valores el 0 o el 1 , el cubit puede tomar ambos valores al mismo tiempo, lo cual permite realizar una cantidad mayor de operaciones a la vez (Textos científicos.com, 2014).

Para dar un ejemplo de la diferencia de capacidad que ostenta, citare el caso de Google, quien afirmara haber alcanzado el 23 de octubre de 2019 la supremacía cuántica ${ }^{3}$. La misma habría consistido en haber logrado realizar tan solo en 3 minutos y 20 segundos un cálculo que con la tecnología a la cual estamos acostumbrados demandaría nada menos

\footnotetext{
${ }^{2}$ Los ordenadores cuánticos se basan en el uso de los cubits (bits cuánticos) en lugar de bits, y da lugar a nuevas puertas lógicas que hacen posibles nuevos algoritmos. Poseen una capacidad de cálculo muy superior a los computadores actuales gracias al paralelismo masivo (exponencial) debido a la superposición de estados en los cubit. En el campo de la criptografía proponen un nuevo enfoque: control absoluto de seguridad a nivel de comunicación y su capacidad para realizar operaciones de factorización (descomposición en números primos), que representa una amenaza para las comunicaciones encriptadas que emplean muchas instituciones en sus sistemas de seguridad, y que se basan a su vez en la dificultad de hacer códigos. Y decir que la computación cuántica es un campo en el que aún queda mucho por descubrir.

${ }^{3}$ Google y un logro muy importante llamado supremacía cuántica que podría permitir-un poco como lo vengo ya insinuando- que nuevos tipos de computadoras hagan cálculos a velocidades que son inconcebibles con la tecnología actual. El poder de dichos cálculos atribuibles a un ordenador nuevo que habría desarrollado el famoso buscador, el cual requiere de condiciones muy específicas para su mantenimiento.
} 
Galán, S. La Computación Cuántica: nuevos desafíos para el sistema legal argentino Derecho y Ciencias Sociales. Noviembre 2020 - Abril 2021. № 24 .Pgs 123 -135 ISSN 1852-2971. Instituto de Cultura Jurídica y Maestría en Sociología Jurídica. Facultad de Ciencias Jurídicas y Sociales. Universidad Nacional de La Plata. Argentina.

que 10.000 años, todo esto con un ordenador de 54 cubits (Gibney, 2019). Llegado el caso, para dicha experimentación no es la única empresa que se está desarrollando, lo mismo ocurre con IBM quien pudo armar hacia septiembre del año pasado el primer ordenador capaz de operar con 53 qubits y está preparando el primer ordenador cuántico para desarrollar en Alemania, el cual sería el primero dentro de la Unión Europea ${ }^{4}$. Además, otras empresas como Microsoft o Intel se encuentran trabajando en proyectos

A la luz de dichos acontecimientos se podría decir que estamos en una etapa en la cual se están viendo resultados concretos de la computación cuántica. Lo cual generaría un lógico entusiasmo en cualquiera, sin embargo, hay que remarcar que se debe estar a la altura de tales avances, pensando el caso desde el lado de la responsabilidad que genera dicha capacidad.

Teniendo presente la dinámica superior de calculo que manifestaron las distintas compañías poseer, resulta claro que la aptitud de modificar el estatus actual de diversas ciencias e inclusive la tecnología con la que las personas se manejan en su vida cotidiana. Esto aplicaría en primera instancia en lo que el manejo de internet respecta tanto para buscadores, aplicaciones como otra índole de páginas webs y es en ello donde quiero hacer un especial hincapié.

Para tal cuestión, no solo importa el papel que tomen los desarrolladores de los mismos ni los fines con los cuales los hubieran creado, también se suscita un rol clave desde las ciencias del derecho, tanto a niveles locales de los Estados legislando como a nivel internacional de los Estados firmando tratados al efecto.

\section{Usos y compatibilidades de la Computación Cuántica}

Para visibilizar de manera concreta la relevancia que pudiera tener la computación cuántica en nuestra vida cotidiana, pasare a mencionar algunos de los usos que tiene y potencialmente podría tener. Según explica el profesor Neugebauer "La informática cuántica tiene potencial para analizar los sistemas complejos en los negocios y la industria, resolver interacciones moleculares y químicas, solucionar complicados

\footnotetext{
${ }^{4}$ IBM anuncia un nuevo hito dentro del sector de la computación cuántica. Su $14^{\circ}$ ordenador cuántico será el más potente hasta ese momento, siendo capaz de operar con 53 qubits. Un nuevo ordenador que estará disponible en breve para toda la familia de clientes que trabajan con los servicios cuánticos de IBM en la nube.
} 
Galán, S. La Computación Cuántica: nuevos desafíos para el sistema legal argentino Derecho y Ciencias Sociales. Noviembre 2020 - Abril 2021. № 24 .Pgs 123 -135 ISSN 1852-2971. Instituto de Cultura Jurídica y Maestría en Sociología Jurídica. Facultad de Ciencias Jurídicas y Sociales. Universidad Nacional de La Plata. Argentina.

problemas de organización y hacer la inteligencia artificial significativamente más potente" 5 . Lo cual ya se podía inferir al haber remarcado ut supra el aumento exponencial de la capacidad de cálculo tanto como la cantidad de cálculos que en formas simultaneas es capaz de realizar, y de este modo, todo lo que conlleve a ecuaciones, químicas, físicas, matemáticas va de suyo que se simplifica (Miller, 2020).

Así mismo el profesor Martin Delgado encuentra oportunidades en el sector de reparto de bienes y mercaderías, donde según indica que "cuando hay muchos repartidores los algoritmos a veces colapsan"6. Además, como si esto fuera poco, actualmente las empresas de automóviles exploran su uso para la creación de nuevos materiales y para mejorar su tráfico reduciendo tiempos de viajes (Martin Delgado, 2019).

Por último y quizás por su peso sea el dato más importante a considerar, se encuentra el relacionado con la ciberseguridad, en el cual Delgado señala que "hay algoritmos que pueden romper claves de encriptación y otros que sirven para buscar en bases de datos" pero también marca que estos algoritmos necesitan números de cubits muy grandes y aún se buscan aplicaciones más compatibles con su uso actual. Esto se traduce en un muy potente desarrollo en el largo plazo para romper todo tipo de claves o contraseñas, y de ello se desprende la incertidumbre de si vendrá a la par con un sistema de seguridad informática que se encuentre a la altura de dicha circunstancia (Ídem, Martin Delgado, 2019).

Piénsese por un momento la potencia que se encuentra en las manos de quienes desarrollan y poseen estos ordenadores, en una era de cuentas bancarias digitales, criptomonedas, plataformas de compra y venta de productos en línea, de teletrabajo y de almacenamiento de datos sensibles de personas como de empresas en la web. Urge entender esta situación y tomar conciencia de ello tanto como ciudadano, como interprete jurídico de sus posibles efectos y por supuesto que como Estado. Tal apertura a radicales cambios abre posibilidades que en muchos casos ni los mismos desarrolladores pueden hoy imaginar, y resultaría esencial que los Estados puedan adecuarse en tiempo y forma

\footnotetext{
5 Reimund Neugbauer, el Presidente del Instituto de Investigación de Fraunhofer explica los alcances que considera al alcance de la Computación Cuántica

${ }^{6}$ Miguel Ángel Martin-Delgado es Director del grupo de Computación Cuántica de la Facultad de Física de la Universidad Complutense de Madrid. En esta nota nos revela los usos que según sus conocimientos profesionales puede lograr el campo de la tecnología cuántica hasta el momento, así también aquellos campos que cree se pueden desarrollar con la misma.
} 
Galán, S. La Computación Cuántica: nuevos desafíos para el sistema legal argentino Derecho y Ciencias Sociales. Noviembre 2020 - Abril 2021. № 24 .Pgs 123 -135 ISSN 1852-2971. Instituto de Cultura Jurídica y Maestría en Sociología Jurídica. Facultad de Ciencias Jurídicas y Sociales. Universidad Nacional de La Plata. Argentina.

a fin de que los ciudadanos puedan explotar sus efectos de forma positiva en cumplimiento de la ley.

\section{Tomar las riendas del desafío cuántico}

Habida cuenta del crecimiento informático descripto y de su capacidad de alterar varios campos de acción humana distintos, es de notar, que es muy probable que resulten nuevas dificultades a resolver sobre viejos paradigmas de las ciencias jurídicas. Esto es, encontrarse con situaciones conflictivas dignas de litigios, pero que nos encuentren con normas anticuadas no pensadas para la naturaleza de dicho conflicto, mediante las cuales, las interpretaciones que se puedan hacer podrían ser imprecisas o encontrarnos con zonas grises. Es por esto que entiendo, es deber de un Estado incorporar a su agenda el seguimiento de dichos avances y reglar conforme a los principios y garantías de su Constitución, los efectos jurídicos de innovaciones que pudieran superar la imaginación -y por ende voluntad- del legislador al momento de sancionar las distintas normas.

Esta regulación, en principio sería un desafío ante una tecnología tan incipiente, de la cual aún no tenemos claros todos los efectos que se pudieran producir. De todos modos, sería posible -al menos en el corto plazo- ir delineando políticas de protección de datos acordes a los estándares más exigentes de la actualidad, tales como El Reglamento General de Protección de Datos de Europa ${ }^{8}$ (Reglamento (UE) 2016/679, 2016) o la Ley General de Protección de Datos de Brasil ${ }^{9}$ (Ley número 13.709/18). Teniendo en cuenta que nuestra normativa en esa materia data del año 2000, la ley numero 25.326 cuyo nombre es "Ley de Protección de Datos Personales" ${ }^{\prime 10}$ en base a su antigüedad y los avances desarrollados en todo este tiempo en la materia, se puede inferir que muchas de sus disposiciones no se encuentran a la orden del día y así mismo lo confirma la práctica, es por ello que en el presente me encargo de tratar algunos de los supuestos que a mi entender urgen revisar, porque en comparación con el Reglamento Europeo han quedado vetustos.

\footnotetext{
${ }^{8}$ Dicho reglamento es el relativo a la protección de las personas físicas en lo que respecta al tratamiento de datos personales y a la libre circulación de estos datos. El mismo suscita un resguardo de avanzada en consideración al régimen local argentino, pues tiene en cuenta muchas definiciones y relaciones existente en la actualidad y las contempla

${ }^{9}$ La Ley de Protección de Datos de Brasil, presenta un estándar muy nutrido en el cual establece entre otros: la persona del Oficial de protección de datos, las notificaciones de violación de datos, el consentimiento para el procesamiento de datos, requisitos de seguridad y privacidad, requisitos de grabación entre otros.

${ }^{10}$ La Ley de Protección de los Datos Personales data del año 2000 y encuentra grandes vacíos regulatorios, es por ello que recibió intentos de modificaciones entre el 2016 y 2020, pero estos no prosperaron.
} 
Galán, S. La Computación Cuántica: nuevos desafíos para el sistema legal argentino Derecho y Ciencias Sociales. Noviembre 2020 - Abril 2021. № 24 .Pgs 123 -135 ISSN 1852-2971. Instituto de Cultura Jurídica y Maestría en Sociología Jurídica. Facultad de Ciencias Jurídicas y Sociales. Universidad Nacional de La Plata. Argentina.

Es necesario entender que, con el paso del tiempo, surgen nuevos paradigmas de trabajo en el campo de la computación e internet, que en un primer momento no se imaginaban o pensaban desarrollar- al menos en el corto tiempo- y es por esto que conceptos claves como "datos genéticos" o" datos biométricos" no supieron ser atendidos por nuestra ley. También es así con otros conceptos de especial relevancia como "empresa”, "grupo empresarial", "normas cooperativas vinculantes" o "servicio de la sociedad de la información" que el Reglamento Europeo en 2018 se encargó especialmente de tratar.

Otra definición importante a atender es la de datos personales, en la cual la ley argentina no hace ningún tipo de reflexión ni definición y hoy constituye un valor trascendente, puesto que es muy grande la cantidad de datos que las personas suben tanto a sus redes sociales, como a distintos blogs, o al registrarse en páginas de internet de todo tipo y es valioso definirlas e identificarlas para poder tratarlas de un modo seguro y cuidarlas. Por su parte el Reglamento General de Datos de Europa deja establecido que una persona se considerará identificable cuando su identidad pueda "determinarse, directa o indirectamente, en particular mediante un identificador, como por ejemplo un nombre, un número de identificación, datos de localización, un identificador en línea o uno o varios elementos propios de la identidad física, fisiológica, genética, psíquica, económica, cultural o social de dicha persona".

En relación al tratamiento de datos sensibles, también vale aclarar que se encuentran incorporados los datos genéticos y los biométricos al Reglamento General de Datos de Europa, y resultaría muy valioso tenerlo en cuenta para actualizar nuestra ley. Puesto que a la hora de identificar a una persona física de manera univoca, resulta de inmenso valor, por otra parte, el acceso a este tipo de innovaciones ya se encuentra de fácil acceso para el público general, con lo cual las relaciones y usos que se le den requieren su reconocimiento jurídico para poder velar desde el Estado por su buen uso.

Es menester entender también las relaciones de sujetos que se contemplan cuando hablamos de protección de datos, en ello la clasificación argentina es bien simple los divide en:

- El titular de los datos

- El responsable de la base de datos

- El usuario de los datos 
Galán, S. La Computación Cuántica: nuevos desafíos para el sistema legal argentino Derecho y Ciencias Sociales. Noviembre 2020 - Abril 2021. № 24 .Pgs 123 -135 ISSN 1852-2971. Instituto de Cultura Jurídica y Maestría en Sociología Jurídica. Facultad de Ciencias Jurídicas y Sociales. Universidad Nacional de La Plata. Argentina.

Simplemente se encarga de las personas cuyos datos son objeto de tratamiento, de quien los archiva como responsable y de quien pueda realizar un tratamiento de dichos datos. En cambio, en el Reglamento General de Datos de Europa nos encontramos con una clasificación más amplia, a saber:

- El interesado

- El responsable

- El Encargado

- El destinatario

- El tercero

- El representante

El interesado se trata simplemente de la figura que encontramos en nuestra Ley como "titular de datos" y no ofrece ninguna distinción, así como también ocurre con "el responsable". "El encargado" debe decirse que es quien trate datos personales por cuenta del responsable de su tratamiento, en tanto que "el destinatario" es aquel a quien se le comunican datos personales que pudieran ser o no de un tercero, "el tercero" es aquella persona distinta del interesado, del responsable de tratarlos, de su encargado y de las personas autorizadas para tratar los datos personales bajo autoridad del responsable o encargado. Por último, el representante es quien representa al responsable o al encargado en cuanto se trate de obligaciones impuestas por el Reglamento (ACD Por los derechos civiles, res 6 julio 2020). ${ }^{11}$

Ante esto es inminente la necesidad de una reforma, mediante la cual se pueda actualizar y por sobre todo preparar al sistema para transformaciones más profundas. Reformas que ya se pueden prever que generaran nuevas figuras legales a contemplar, o en cuanto menos profundizará esquemas de figuras que ya existen y se encuentran contempladas en la normativa europea pero no en la nuestra, como muestran los casos vistos anteriormente.

Sería de esperar que, en cuanto antes se puedan preparar a nuestros cuerpos legales, más fácil ser interpretar o encasillar las nuevas situaciones fácticas que se puedan suscitar, o mejor dicho existirá una mayor facilidad para poder resolver los nuevos conflictos que de ellas se obtengan. Caso contrario, es muy probable que se genere un vacío legal que

\footnotetext{
${ }^{11}$ En el texto referente se encontrarán las principales comparaciones a las cuales paso a hacer referencia expresamente. En ella se encuentra el móvil primordial para realizar la comparación legislativa respectiva entre el Reglamento General de Protección de Datos de Europa y la Ley de Protección de Datos de Argentina.
} 
Galán, S. La Computación Cuántica: nuevos desafíos para el sistema legal argentino Derecho y Ciencias Sociales. Noviembre 2020 - Abril 2021. № 24 .Pgs 123 -135 ISSN 1852-2971. Instituto de Cultura Jurídica y Maestría en Sociología Jurídica. Facultad de Ciencias Jurídicas y Sociales. Universidad Nacional de La Plata. Argentina.

ocasione en los operadores judiciales una incertidumbre supina, ya que, lejos de referenciar y mostrar cómo debería resolver la autoridad, podría la ausencia de especificidad aumentar el margen de discrecionalidad para resolver de los magistrados. Dicha situación caería en la consecuencia de generar una nueva faz de inseguridad jurídica, lo cual sería contraproducente para explorar y explotar el potencial de la tecnología cuántica en nuestro país. De mas esta decir la importancia que posee que todo ciudadano tenga certezas, sobre los márgenes de legalidad que poseen sus actos.

\section{Avances en tecnología cuántica de los Estados}

En torno a la utilización de la nueva tecnología de computación cuántica, es menester señalar que existe una carrera entre los distintos países por ver quien consigue desarrollar más rápida y óptimamente estos avances de la ciencia. No es de extrañar que se despierte este interés en ciertas cuestiones delicadas para los Estados como pueden ser la seguridad nacional, la protección de datos, los servicios de inteligencia o así mismo la protección de las cuentas bancarias y finanzas públicas.

Ante esta situación se pueden nombrar diversos proyectos muy ambiciosos, en los cuales se viene trabajando en los últimos años. Un salto muy importante de la tecnología cuántica fue realizado por China en el año 2016, año en el cual lanzo al espacio el primer satélite cuántico, el satélite Minicius ${ }^{12}$.

El satélite Minicius cuya sigla oficial es" QUESS", tiene como principal objetivo crear redes de comunicación a prueba de hackeos a nivel global, dicha meta no era inédita en cuanto a su función de cifrado, pero sí lo era para una extensión a nivel global de la misma. Lo cual despierta el interés de las agencias de seguridad, redes de espionaje y gobiernos más importantes (BBC MUNDO, 2016).

Otro proyecto muy importante se está transitando en Nueva York se trata del cable cuántico de seguridad, el mismo se representa con las siglas “QKD” y se utiliza para la protección de los datos de los bancos y grandes empresas. Este cable viene a reemplazar a la fibra óptica tradicional, la cual es susceptible de ser vulnerada y reviste un cambio

\footnotetext{
${ }^{12}$ El proyecto Minicius se trata del ambicioso proyecto chino por domar antes que nadie la tecnología satelital cuántica. El satélite QUESS tiene como principal objetivo crear nuevas redes de comunicación globales a prueba de hackeos. Se trata de una tecnología revolucionaria en la comunicación en los campos militares, de gobierno y finanzas. Por eso, este millonario proyecto es seguido de cerca no sólo por la comunidad científica, sino también por las agencias de seguridad y espionaje del mundo.
} 
Galán, S. La Computación Cuántica: nuevos desafíos para el sistema legal argentino Derecho y Ciencias Sociales. Noviembre 2020 - Abril 2021. ํo 24 .Pgs 123 -135 ISSN 1852-2971. Instituto de Cultura Jurídica y Maestría en Sociología Jurídica. Facultad de Ciencias Jurídicas y Sociales. Universidad Nacional de La Plata. Argentina.

radical, puesto que, según su empresa desarrolladora Quantum Exchange, posee propiedades cifrado irrompibles aun para la tecnología cuántica en curso. Dicha empresa deja en claro que su misión es darle a las empresas comerciales y agencias gubernamentales el mejor y más innovador sistema de defensa para mantener sus datos seguros hoy y en el futuro, ${ }^{13}$ dejando entrever que se está preparando a los sistemas de transmisión de datos, para poder defenderse sin sobresaltos de distintos ataques cibernéticos tanto a una empresa, como a un Estado o un individuo, lo cual constituye un pilar fundamental de seguridad jurídica.

Vale recalcar que la profundidad de este proyecto de encriptación tan novedoso, está siendo pensado y proyectado para sistemas de quebrantamiento de seguridad de poder cuántico, pero aun así sería necesario esperar que los mismos estén en marcha intentando vulnerarlo para saber qué tan resistente realmente es (Liao, S., Cai, W., Liu, 2017).

Por el lado de la Unión Europea se encuentra el proyecto "OpenSuperQ"14 el mismo cuenta con la participación de varios países como Alemania, Suiza, Suecia e Irlanda y se trata de un proyecto de interés meramente de desarrollo científico sin objetivos económicos. El mismo consiste en el desarrollo de una base de datos para la investigación científica a la cual todos puedan tener acceso, en una suerte de "democratización" del acceso a la información de investigaciones que pudieran ser muy avanzadas (Lamata, 2019).

Si bien varían las naciones que encuentran ambiciosos proyectos de distinta índole para realizar, dista Latinoamérica aun de formar parte de dicho selecto grupo, y es allí donde entra la necesidad de empezar a delinear algún tipo de estrategia desde nuestro lugar para que las mismas no nos encuentren desprevenidos o a la deriva. Vale decir que para cuyo

\footnotetext{
${ }^{13}$ El túnel Holland, que conecta la isla de Manhattan, en Nueva York, con el Estado de Nueva Jersey, es uno de los más antiguo de los destinados al tráfico de vehículos en Estados Unidos. Fue inaugurado en 1927, pero bajo su vieja estructura se esconde una nueva tecnología que va camino de convertirse en el futuro de las telecomunicaciones. En ese túnel se ocultan unos cables de fibra óptica hechos con conocimientos de mecánica cuántica, que tienen la capacidad de proteger datos críticos de bancos y agencias gubernamentales. Se trata de una tecnología llamada sistema de distribución de claves cuánticas $(\mathrm{QKD})$ que permite generar e intercambiar claves secretas usando dos fotones entrelazados que solo conocen emisor y receptor, la finalidad principal seria poder generar un intercalado de claves cuando una de estas es vulnerada, palabras más palabras menos y así obtener un sistema de seguridad más eficiente

${ }^{14}$ El proyecto OpenSuperQ combina el desarrollo de hardware (los circuitos superconductores) para construir un chip con entre 50 y 100 cúbits, con la creación de software, es decir, de algoritmos cuánticos de alto nivel y su descomposición en secuencias de pulsos para actuar sobre el chip y producir las secuencias de puertas lógicas cuánticas que son necesarias. Entre los campos de aplicación de los futuros ordenadores cuánticos figuran el diseño de materiales, las reacciones químicas, las finanzas, los problemas de optimización y el famoso "machine learning"
} 
Galán, S. La Computación Cuántica: nuevos desafíos para el sistema legal argentino Derecho y Ciencias Sociales. Noviembre 2020 - Abril 2021. № 24 .Pgs 123 -135 ISSN 1852-2971. Instituto de Cultura Jurídica y Maestría en Sociología Jurídica. Facultad de Ciencias Jurídicas y Sociales. Universidad Nacional de La Plata. Argentina.

fin es relevante un tratamiento no solo desde lo estrictamente académico en las distintas universidades y centros de estudio de la computación, sino también una visión transversal que pueda llevarse a cabo en las distintas ciencias, y por supuesto que la ciencia jurídica no escapa a esto.

\section{Conclusión}

En mi opinión resultaría una equivocación no prever que estos procesos además de poder ser grandes creadores de soluciones a problemas actuales, podrían generar pérdidas muy potentes en caso de no caer en manos adecuadas o de no poseer cierta protección adecuada. Es por esto que ante el escenario nuevo el mundo del derecho no es ajeno, y más allá de que los principales desarrollos de esta tecnología incipiente recién se están vislumbrando en los países más desarrollados, también es cierto que el internet es justamente una red mundial de la cual nadie puede esconderse.

En otras palabras, lo que justamente se produce a miles de kilómetros, trátese de China, Europa o Estados Unidos puede tener un efecto inmediato en cualquier computadora o programa que se esté usando en Argentina, sobre todo teniendo presente que existe una potencia muy grande para romper sistemas de seguridad que actualmente poseen todas nuestras páginas web y aplicaciones.

Es realmente alarmante para la realidad en la cual nos encontramos, el hecho de que ni siquiera podamos aprobar alguna ley para estar a la altura de los últimos desarrollos tecnológicos, de lo cual me refiero puntualmente a la Ley de Datos y la importancia de proteger un universo virtual que era inimaginable en el año 2000 cuando la misma fue sancionada.

La Democracia tiene una deuda insoslayable con las regulaciones tecnológicas en Argentina y eso sin hablar de aspirar a desarrollar proyectos del tipo que se producen en China o Estados Unidos a los cuales no tendremos acceso hasta dentro de un futuro que hoy es incierto.

Por lo pronto existen ciertas situaciones que se pueden atender, nos encontramos a tiempo de producir un marco de protección para las personas, empresas y Estado de modo transversal, conceptualizando de modo legal y como respectivamente corresponda las nociones básicas de la computación cuántica. 
Galán, S. La Computación Cuántica: nuevos desafíos para el sistema legal argentino Derecho y Ciencias Sociales. Noviembre 2020 - Abril 2021. № 24 .Pgs 123 -135 ISSN 1852-2971. Instituto de Cultura Jurídica y Maestría en Sociología Jurídica. Facultad de Ciencias Jurídicas y Sociales. Universidad Nacional de La Plata. Argentina.

Esta tarea podría venir de la mano con anticipar discusiones en los distintos ámbitos académicos y llevar un especial énfasis en seguir de cerca las problemáticas que se plantean en los distintos proyectos del mundo, para que cuando llegue la posibilidad de incorporarlas a un país como el nuestro, no repitamos esos mismos errores.

\section{Bibliografía}

Ferreyra, E. ( S/F) El RGPD y la ley argentina de protección de datos personales Análisis comparativo. https://adc.org.ar/wp-content/uploads/2019/06/041-el-RGPD-y-la-leyargentina-de-proteccion-de-datos-personales-XX-2018.pdf

BBC Mundo (17/08/2016), Minicius, el pionero satélite cuántico chino que puede revolucionar la historia de las comunicaciones del mundo. https://www.bbc.com/mundo/noticias-37064468

Darwish Miranda, N. (S/D). Computación Cuántica. Universidad de La Laguna. https://www.mundotec.com.ar/Computacion-Cuantica.pdf

Gibney, E. ( 23 de octubre de 2019) Google Publishes Landmark Quantum Supremacy Claim. Scientific American .https://www.scientificamerican.com/article/googlepublishes-landmark-quantum-supremacy-claim/

Edwards, J.(16 de mayo de 2019). Entendiendo La Ley General de Protección de Datos de Brasil, Progress formerly ipswitch. https://blog.ipswitch.com/es/entendendiendola-ley-general-de-proteccion-de-datos-en-brasil

Lamata, L. (23 de enero de 2019) .Así será OpenSuperQ, el ordenador cuántico más avanzado de Europa. Nmas1.org https://nmas1.org/news/2019/01/24/opensuperqcuantico-ciencia

Ley $\mathrm{N}^{\mathrm{o}} 25326$ de Protección de los Datos Personales (Argentina) https://www.oas.org/juridico/PDFs/arg_ley25326.pdf

Liao, SK., Cai, WQ., Liu, WY. et al. Satellite-to-ground quantum key distribution. Nature 549, 43-47 (2017). https://doi.org/10.1038/nature23655

Delgado, M (5 de abril de 2019) ¿Para qué sirve un ordenador cuántico? BBVA.Argentina. https://www.bbva.com/es/para-que-sirve-un-ordenador-cuantico/

Miller, ( 16 de Marzo de 2020) IBM prepara el primer ordenador cuántico de la UE, Expansión Economía Digital. https://www.expansion.com/economiadigital/companias/2020/03/16/5e6eb6bae5fdea724b8b45a3.html

Diario Oficial de la Unión Europea (2016) Reglamento (UE) 2016/679 del Parlamento europeo y del Consejo de 27 de abril de 2016 relativo a la protección de las personas 
Galán, S. La Computación Cuántica: nuevos desafíos para el sistema legal argentino Derecho y Ciencias Sociales. Noviembre 2020 - Abril 2021. № 24 .Pgs 123 -135 ISSN 1852-2971. Instituto de Cultura Jurídica y Maestría en Sociología Jurídica. Facultad de Ciencias Jurídicas y Sociales. Universidad Nacional de La Plata. Argentina.

físicas en lo que respecta al tratamiento de datos personales y a la libre circulación de estos datos y por el que se deroga la Directiva 95/46/CE (Reglamento general de protección de datos). https://www.boe.es/doue/2016/119/L00001-00088.pdf

Sanz, R.( 19 de septiembre de 2019). IBM consigue un nuevo récord en la computación cuántica, el primer ordenador comercial de 53 qubits. Computerhoy.com.:https://computerhoy.com/noticias/tecnologia/ibm-computacioncuantica-primer-ordenador-comercial-53-qubits-495027

Textoscientificos.comn (1 de septiembre 2014) Computación Cuántica. https://www.textoscientificos.com/node/1880 